\title{
KIT is involved in melanocyte proliferation, apoptosis and melanogenesis in the Rex Rabbit
}

\author{
Shuaishuai Hu ${ }^{1}$, Yang Chen ${ }^{1,2}$, Bohao Zhao ${ }^{1}$, Naisu Yang ${ }^{1}$, Shi Chen ${ }^{1}$, Jinyu Shen ${ }^{1}$, Guolian Bao ${ }^{3}$, Xinsheng Wu \\ Corresp. 1, 2 \\ ${ }^{1}$ College of Animal Science and Technology, Yangzhou University, Yangzhou, Jiangsu, China \\ 2 Joint International Research Laboratory of Agriculture \& Agri-Product Safety, Yangzhou University, Yangzhou, Jiangsu, China \\ 3 Animal Husbandry and Veterinary Research Institute, Zhejiang Academy of Agricultural Sciences, Hangzhou, Zhejiang, China \\ Corresponding Author: Xinsheng Wu \\ Email address: xswu@yzu.edu.cn
}

Background: Melanocytes play an extremely important role in the process of skin and coat colors in mammals which is regulated by melanin-related genes. Previous studies have demonstrated that KIT is implicated in the process of determining the color of the coat in Rex rabbits. However, the effect of KIT on the proliferation and apoptosis of melanocytes and melanogenesis has not been clarified. Methods: The mRNA and protein expression levels of KIT were quantified in different coat colored rabbits by qRT-PCR and a Wes assay. To identify whether KIT functions by the regulation of melanogenesis, KIT overexpression and knockdown was conducted in melanocytes, and KIT mRNA expression and that of the melanin-related genes TYR, MITF, PMEL and DCT were quantified by qRTPCR. To further confirm whether KIT influences melanogenesis in melanocytes, the levels of melanin were quantified using $\mathrm{NaOH}$ lysis after overexpression and knockdown of KIT. Melanocyte proliferation was estimated using a CCK-8 assay at 0, 24, 48 and $72 \mathrm{~h}$ after transfection, and the rate of apoptosis of melanocytes wasmeasured by fluorescenceactivated cell sorting. Results: KIT mRNA and protein expression levels were significantly different in the skin of Rex rabbits with different color coats $(P<0.05)$, the greatest levels observed in those with black skin. The mRNA expression levels of KIT significantly affected the mRNA expression of the pigmentation-related genes TYR, MITF, PMEL and DCT $(P<$ $0.01)$. Melanin content was evidently regulated by the change in expression patterns of KIT $(P<0.01)$. In addition, KIT clearly promoted melanocyte proliferation, but inhibited apoptosis. Conclusions: Our results reveal that KIT is a critical gene in the regulation of melanogenesis, controlling proliferation and apoptosis in melanocytes, providing additional evidence for the mechanism of pigmentation of animal fur. 


\section{$1 \quad$ KIT is involved in melanocyte proliferation, apoptosis and}

\section{melanogenesis in the Rex Rabbit}

3 Shuaishuai $\mathrm{Hu}^{1}$, Yang Chen ${ }^{1,2}$, Bohao Zhao ${ }^{1}$, Naisu Yang ${ }^{1}$, Shi Chen ${ }^{1}$, Jinyu Shen ${ }^{1}$, Guolian

$4 \mathrm{Bao}^{3}$, Xinsheng $\mathrm{Wu}^{1,2 *}$

$5{ }^{1}$ College of Animal Science and Technology, Yangzhou University, Yangzhou, 225009,

6 Jiangsu, China

$7 \quad 2$ Joint International Research Laboratory of Agriculture \& Agri-Product Safety, Yangzhou

8 University, Yangzhou, 225009, Jiangsu, China

$9{ }^{3}$ Animal Husbandry and Veterinary Research Institute, Zhejiang Academy of Agricultural

10 Sciences, Hangzhou 310021, Zhejiang, China

11

$12 *$ Corresponding author:

13 Xinsheng $\mathrm{Wu}$

1448 South University Ave., Yangzhou, Jiangsu 225009, P. R. China

15 E-mail: xswu@yzu.edu.cn 
16

17

\section{Abstract}

Background: Melanocytes play an extremely important role in the process of skin and coat colors in mammals which is regulated by melanin-related genes. Previous studies have demonstrated that KIT is implicated in the process of determining the color of the coat in Rex rabbits. However, the effect of KIT on the proliferation and apoptosis of melanocytes and melanogenesis has not been clarified.

Methods: The mRNA and protein expression levels of KIT were quantified in different coat colored rabbits by qRT-PCR and a Wes assay. To identify whether KIT functions by the regulation of melanogenesis, KIT overexpression and knockdown was conducted in melanocytes, and KIT mRNA expression and that of the melanin-related genes TYR, MITF, PMEL and DCT were quantified by qRT-PCR. To further confirm whether KIT influences melanogenesis in melanocytes, the levels of melanin were quantified using $\mathrm{NaOH}$ lysis after overexpression and knockdown of KIT. Melanocyte proliferation was estimated using a CCK-8 assay at 0, 24, 48 and $72 \mathrm{~h}$ after transfection, and the rate of apoptosis of melanocytes wasmeasured by fluorescenceactivated cell sorting.

Results: KIT mRNA and protein expression levels were significantly different in the skin of Rex rabbits with different color coats $(P<0.05)$, the greatest levels observed in those with black skin. The mRNA expression levels of KIT significantly affected the mRNA expression of the pigmentation-related genes TYR, MITF, PMEL and DCT $(P<0.01)$. Melanin content was evidently regulated by the change in expression patterns of KIT $(P<0.01)$. In addition, KIT clearly promoted melanocyte proliferation, but inhibited apoptosis.

Conclusions: Our results reveal that KIT is a critical gene in the regulation of melanogenesis, controlling proliferation and apoptosis in melanocytes, providing additional evidence for the mechanism of pigmentation of animal fur.

Keywords: KIT; Melanocyte; Melanogenesis; Proliferation; Apoptosis 


\section{Introduction}

Rex rabbits have an important and archetypal fur, which is represented in China by a considerable number of colors, including black, white, chinchilla, brown, gray, gray-yellow, etc. (Figure 1). Therefore, it serves as an important experimental animal model of pigmentation, which is instructive in defining mammalian coat colors. The diverse range of hair and skin colors in mammals can be attributed to the type and quantity of pigment. However, the formation of pigment is regulated by the interaction of different genes and environmental factors (Sturm et al., 2001). Melanin produced by melanocytes is one of a number of pigments that are regulated by melaninrelated genes, which determine the production of phenotypes of diverse hair and skin color in mammals. Currently, there are 128 genes of pigmentation associated with phenotypes in humans (Baxter et al., 2019). More than 170 pigmentation-related genes associated with coat color phenotype have been identified in mice (Bennett \& Lamoreux 2003). Moreover, at least 31 genes that have a significant effect on coat color have been identified in mink (Song et al., 2017). Therefore, it might be reasonable to conclude that the coat color of Rex rabbits is regulated by various pigmentation-related genes.

Among melanin-related genes, KIT is pivotal in the melanogenesis signaling pathway, and mutations or deletion of KIT can cause different hair and skin colors in mammals. KIT is a protooncogene, classified as a member of the tyrosine kinase receptor family, the product of which is the type III tyrosine kinase. In addition, KIT plays an important role in the melanogenesis pathway. It was reported that melanogenesis can be enhanced by stem cell factor/c-kit signaling in normal human epidermal melanocytes exposed to norepinephrine (Lan et al., 2008). In previous studies, the occurrence of melanomas was found to be often associated with a mutation in KIT (Curtin et al., 2006; Sakaizawa et al., 2015). It has been reported that a single $A \rightarrow G$ base missense mutation in exon 13 causes differential expression of KIT in Liaoning Cashmere goats resulting in different coat colors (Li et al., 2017). The duplication of chromosome 6 and aberrant insertion on chromosome 29 leads to coat color variations in white Galloway cattle and white Park cattle (Brenig et al., 2013). White spotting is caused by a frameshift mutation of KIT in the Arabian camel (Holl et al., 2017). Furthermore, melanogenesis is additionally regulated by a number of key genes, which control melanocytes to further generate melanin, such as the key melanogenic enzyme genes TYR and DCT (Hearing 2011; Yasumoto et al., 1997), and critical melanosomal protein PMEL (Chen et al., 2018; Ji et al., 2018). In addition, KIT has a critical influence on melanin deposition. It is known that KIT regulates cell migration, survival, proliferation and differentiation in melanocytes (Garrido \& Bastian 2010; Grichnik 2006) and interacts with MITF, a crucial gene that is implicated in the formation of melanin and further regulates the development of melanocytes (Mizutani et al., 2010; Reiko et al., 2004; Wen et al., 2010). Our previous studies 
have indicated that KIT is involved in the formation of different coat colors in the Rex rabbit. However, the role of KIT in melanogenesis, proliferation and apoptosis in melanocytes remains largely unexplored. Furthermore, it is necessary to determine whether the expression of KIT can indirectly affect melanogenesis-related genes.

In the present study, the mRNA expression levels of KIT and melanin-related genes in diverse coat colors of the Rex rabbit were measured by quantitative real-time PCR (qRT-PCR). In order to reveal the role of KIT in melanocyte proliferation, apoptosis and melanogenesis, the change in rabbit melanocytes was detected by overexpression and knockdown of KIT. The results of the study provide an important theoretical basis for the additional study of mammal skin and hair color.

\section{Materials and methods}

\section{Ethics statement}

This study was conducted in accordance with the recommendations of the Animal Care and Use Committee at Yangzhou University (Jiangsu, China), who approved the experimental procedures (Yangzhou, China, 24 November 2018, No. 201810025) and were strictly implemented according to the regulations for experimental animals.

\section{Experimental animals and sample collection}

Animals were maintained in an ordinary housing facility in keeping with the national standard Laboratory Animal Requirements of Environment and Housing Facilities (GB 14925-2001). The care of laboratory animals and the experimental animal surgery conformed to the Jiangsu Administration Rule of Laboratory Animals. The rabbits were anesthetized using an intraperitoneal injection of sodium pentobarbital. Rex rabbits (black, white, chinchilla, brown, gray and gray-yellow) were provided by Zhejiang Yuyao Xinnong Rabbit Industry Co., Ltd. Four rabbits (male and female in equal numbers) of each coat color were raised within the same environment. Ninety days after birth, dorsal skin samples of identical size $\left(1 \mathrm{~cm}^{2}\right)$ and anatomical location were harvested from each rabbit for RNA and protein extraction. The wounds were treated topically with iodophor after sample collection. All rabbits made a full recovery from surgery and were free to move after two hours.

\section{Cloning of the KIT gene}

Primers for three specific 5' untranslated regions (UTRs), coding sequence (CDS), and two 3' UTRs of KIT were synthesized (Table 1). A rapid amplification of cDNA ends (RACE) assay was conducted in accordance with the kit instructions (Invitrogen \& Clontech, Carlsbad, CA, USA). The coding sequence of the KIT gene was acquired based on the results of 5' and 3' RACE assays. KIT cDNA was cloned and reconstructed into pcDNA3.1 or pcDNA3.1-Myc vectors with 
110

111

112

113

114

115

QuickCut Restriction Enzyme Hind III and EcoR I (Takara, Beijing, China) using a ClonExpress II One Step cloning kit (Vazyme, Nanjing, China), respectively.

\section{Rabbit melanocyte culture and overexpression transfection of KIT}

Rabbit melanocytes were isolated from the dorsal skin tissue of Rex rabbits as described by Chen et al. (Chen et al., 2019). The cells were cultured in M254 medium (Gibco, Carlsbad, CA, USA) supplemented with 1\% human melanocyte growth supplement-2 (HMGS-2, Gibco, Carlsbad, CA, USA) and maintained in an incubator at $37^{\circ} \mathrm{C}$ in an atmosphere containing $5 \% \mathrm{CO}_{2}$. The seeded cells were cultured in 6-well plates to $70-90 \%$ confluence, then overexpression of KIT achieved using Lipofectamine ${ }^{\mathrm{TM}} 3000$ Reagent (Invitrogen, Carlsbad, CA, USA), in accordance with the manufacturer's instructions. A $2 \mu \mathrm{g}$ quantity of KIT DNA plasmid in Opti-MEM ${ }^{\mathrm{TM}}$ medium (Gibco, Carlsbad, CA, USA) was added to $6 \mu$ Lipofectamine $^{\mathrm{TM}} 3000$ diluted with Opti-MEM ${ }^{\mathrm{TM}}$ then incubated for 10-15 mins at room temperature. The DNA-lipid complex was added cells and incubated for $48 \mathrm{~h}$ with M254 medium, prior to qRT-PCR analysis.

\section{Knockdown of KIT using siRNA}

siRNAs of KIT and the negative control were purchased from Shanghai GenePharma Co., Ltd (Table 2). The cells were grown to $70-90 \%$ confluence, then knockdown of KIT was conducted using Lipofectamine 3000 (Invitrogen, Carlsbad, CA, USA), in accordance with the manufacturer's instructions. $0.264 \mu \mathrm{g}(1 \mu \mathrm{L})$ siRNA-KIT and $3 \mu \mathrm{L}$ Lipofectamine 3000 were used in each well of 6-well plates. The transfection method was the same as that used for overexpression transfection of KIT. Transfected cells were analyzed using qRT-PCR after $36 \mathrm{~h}$.

\section{RNA isolation and quantitative real-time PCR (qRT-PCR)}

Total RNA was isolated from skin tissues and transfected cells using an RNAsimple Total RNA kit (Tiangen Biotech (Beijing) Co., Ltd.). The quality and concentrations of RNA samples were quantified from measurements of optical density (OD) (OD=260/280) using a NanoDrop 1000 (ThermoFisher Scientific, New York, USA). One $\mu$ g total RNA was used to synthesize cDNA using a Super RT cDNA kit (Tiangen Biotech Co., Ltd.). Quantitative real-time PCR was conducted using ChamQ ${ }^{\mathrm{TM}} \mathrm{SYBR}^{\circledR}$ qPCR Master Mix (Vazyme, Nanjing, China), with $1 \mu \mathrm{L}$ cDNA in each well and $1 \mu \mathrm{L}$ primer with a $0.5 \mu \mathrm{mol}$ primer final concentration. Data were analyzed using QuantStudio ${ }^{\circledR} 5$ software (Applied Biosystems; Thermo Fisher Scientific, Foster City, CA). Each sample was measured four times and relative expression of target genes computed using the $2^{-\Delta \Delta \mathrm{Ct}}$ method (Schmittgen \& Livak 2008) after normalization to GAPDH as the endogenous control. KIT, MITF, TYR, DCT, PMEL and GAPDH gene sequences were obtained from NCBI for design of the primers (Table 3).

\section{Isolation of proteins and Wes}


Skin and cell lysates were obtained using RIPA lysis buffer (PPLYGEN, Beijing, China). Protein concentrations were quantified using an enhanced BCA protein assay kit (Beyotime, Shanghai, China). A $7.5 \mu \mathrm{g}$ protein sample in each well was analyzed using a Wes automated Western blotting system purchased from Protein Simple (Harris 2015). Analysis was conducted using an anti-GAPDH mouse monoclonal antibody diluted 1:100 (Abcam, Cambridge, UK) and anti-KIT mouse monoclonal antibody (BBI, Beijing, China) diluted 1:100.

\section{Melanin content measurement}

Rabbit melanocytes were obtained $72 \mathrm{~h}$ after transfection then washed in phosphate buffered saline (PBS) (HyClone, Logan, USA) 3 times, then centrifuged at $1000 \mathrm{rpm}$ for $10 \mathrm{~min}$ at $4^{\circ} \mathrm{C}$, the supernatant discarded after each wash. The cells were then lysed in $1 \mathrm{~mL}$ of $1 \mathrm{~mol} / \mathrm{L} \mathrm{NaOH}$ and incubated at $80^{\circ} \mathrm{C}$ for $1 \mathrm{~h}$. Finally, the melanin concentration was determined from measurements of optical density (OD) at $475 \mathrm{~nm}$ using an Infinite M200 Pro (Tecan, Männedorf, Switzerland) spectrophotometer.

\section{Cell proliferation assay}

Cell proliferation was evaluated using a Cell Counting Kit-8 assay (Vazyme, Nanjing, China), in accordance with the manufacturer's instructions, as described previously by Zhao et al. (Zhao et al., 2019). Cells were harvested and seeded in 96-well plates after $16 \mathrm{~h}$ transfection, then the OD at $450 \mathrm{~nm}$ of each well was measured at 0, 24, 48 and $72 \mathrm{~h}$ using an Infinite M200 Pro spectrophotometer (Tecan, Männedorf, Switzerland).

\section{Apoptosis assay}

Apoptosis was measured using an Annexin V-FITC apoptosis detection kit (Vazyme, Nanjing, China). The cells were collected $48 \mathrm{~h}$ after transfection and sorted by fluorescence-activated cell sorting using a FACSAria SORP flow cytometer (Becton Dickinson, San Jose, CA). Each sample was measured in triplicate. Positive FITC staining fluoresces green and PI fluoresces red. Thus, living cells have only very low background fluorescence. Early apoptotic cells have strong green fluorescence only while late apoptotic cells exhibit dual red and green fluorescence.

\section{Statistical analysis}

Each experiment was performed in triplicate and analyzed using IBM SPSS v25 (SPSS Inc., Chicago, IL). Significant differences in relative gene expression were analyzed by one-way ANOVA. All values represent means \pm standard deviation (SD).

\section{Results}

Expression of KIT in the skin of Rex rabbits with different colors of coat 
176

177

178

179

180

181

182

183

184

185

186

187

188

189

190

191

192

193

194

195

196

197

198

199

200

201

202

203

204

205

206

207

208

The 5' UTR and 3' UTR sequences of the KIT gene were acquired using a RACE assay. The coding sequence of KIT was spliced according to the 5' and 3' RACE results, and the KIT cDNA sequence successfully cloned and reconstructed into a pcDNA3.1 or pcDNA3.1-Myc vector (Figure 2a, b and c). The rabbit KIT gene included a 48 bp 5'UTR, 1399 bp 3'UTR and a 2910 bp coding sequence (CDS) which was submitted to NCBI (GenBank: KY971605), and the coding sequence (CDS)of rabbit KIT RACE results was less 12 bp (4 amino acids) than the reference sequence. However, other amino acids are same, and the conserved domains are also same. The phylogenetic relationship of the assembled cDNA of KIT in Oryctolagus cuniculus to other species (Urocitellus parryii, Marmota, Jaculus jaculus, Microtus ochrogaster, Carlito syrichta and Ochotona princeps) was explored using MEGA6. The results indicated that the other six species were divided into two clades, indicating that Oryctolagus cuniculus had a more remote relationship to all other species tested than they did among themselves (Figure 2d).

Subsequently, the expression levels of KIT were quantified in the different coat colors. The mRNA expression of the KIT gene was highest in rabbits with black skin, followed by chinchilla, brown, gray, gray-yellow, the lowest level observed in white skin $(P<0.05)$ (Figure 2e). It was found that the protein expression levels of KIT with black skin were also higher than in rabbits with other skin colors, the lowest being white skin. KIT protein expression was in accordance with that of mRNA expression (Figure 2f).

\section{Melanogenesis-related genes were regulated by overexpression and knockdown of KIT}

The homology of KIT in Oryctolagus cuniculus was compared with other mammals using an NCBI conserved domain search. Approximately $80 \%$ homology was found overall, the rabbit KIT protein possessing a common domain (PKC-like superfamily) that was the same as other mammals (Figure 3a). It is known that PKC- $\beta$ can regulate the activity of tyrosinase, combine with melanin in the cell membrane and further promote melanogenesis (MochlyRosen 1995). To identify whether KIT can regulate melanogenesis, overexpression and knockdown of KIT was performed in melanocytes. The results indicate that knockdown of KIT using three different siRNAs resulted in expression clearly lower than that of the negative control (NC) group $(P<0.01)$, with siRNA791 having the greatest effect (Figure 3b). KIT mRNA and protein expression levels increased significantly after overexpression of KIT $(P<0.01)$, and decreased after knockdown of KIT $(P<$ 0.01) (Figure 3d). Furthermore, KIT mRNA expression and that of the melanin-related genes TYR, MITF, PMEL and DCT increased considerably when KIT was overexpressed in melanocytes (Figure 3f), and decreased substantially after knockdown of KIT $(P<0.01)$ (Figure 3e).

KIT promoted melanogenesis and affected proliferation and apoptosis of melanocytes 
209 To further confirm whether KIT affected melanogenesis in melanocytes, the level of melanin was 210 quantified after both overexpression and knockdown of KIT. Melanin levels increased 211 significantly compared with the control group after overexpression of KIT $(P<0.05)$. Conversely 212 melanin levels clearly decreased (Figure 4a, b). The proliferation of melanocytes was estimated 213 using a CCK-8 assay 0, 24, 48 and $72 \mathrm{~h}$ after transfection. The results indicate that overexpression 214 of KIT greatly promoted melanocyte proliferation from 24 to $72 \mathrm{~h}(P<0.01)$, while proliferation 215 was significantly inhibited after knockdown of KIT $(P<0.01)$ (Figure 4c, d). Furthermore, the 216 degree of melanocyte apoptosis was detected by fluorescence-activated cell sorting. It was found 217 that melanocyte apoptosis was inhibited by KIT overexpression (Figure 4e, f), but knockdown of 218 KIT promoted apoptosis (Figure 4g, h), indicating that KIT had a negative effect on apoptosis.

\section{Melanin content and the expression of KIT have a positive correlation}

220 To determine whether melanin content has correlation with the expression of KIT, correlation analysis was performed by Pearson Correlation in SPSS v. 22.0 software. The results clearly found a significantly positive correlation between the melanin content and the mRNA expression of KIT $(\mathrm{P}<0.01)$ (Table 4). It was demonstrated that the expression of KIT has an important effect on melanin content.

225

226

227

228

229

230

231

232

233

\section{Discussion}

Melanin produced by melanocytes controls mammalian coat color. Differing coat colors represent a high research value and are an economically valuable trait in mammals. Different coat pigmentation is controlled by the ratio of pheomelanin to eumelanin (Greg B \& George C 2007; $\mathrm{N}$ et al., 2013), the former principally involved in the production of red and yellow and the latter participating in the formation of black pigment (Ito et al., 2010; Ito 2010; Shosuke et al., 2010). It has been demonstrated that excess pheomelanin can cause nevus depigmentosus that leads to yellow hair color (Oiso et al., 2018). Reduced pheomelanin results in the formation of brown hair (Ito et al., 2017; Oiso et al., 2018). Previous research has reported that black pigment decreases when eumelanin synthesis is blocked, but the range of red and yellow pigment has been shown to be enhanced (Hirobe et al., 2007; Silvers 1979; Tamate \& Takeuchi 2010). Therefore, we speculated that varying the ratio of pheomelanin to eumelanin plays an important role in the formation of different coat colors in the Rex rabbit.

In the present study, we found that KIT mRNA and protein expression levels were significantly different in the skin of Rex rabbits with different coat colors, the highest observed in black skin, the least in white skin. The results indicate that the ratio of pheomelanin to eumelanin was affected by the expression of KIT, which induced the synthesis of eumelanin. However, KIT mRNA and protein expression levels were not significantly different between a gray and gray- 
243

244

245

246

247

248

249

250

251

252

253

254

255

256

257

258

259

260

261

262

263

264

265

266

267

268

269

270

271

272

273

274

275

yellow color, indicating that the formation of these two coat colors might not correlate with the synthesis of eumelanin, but instead be determined by pheomelanin. Furthermore, we further confirmed that KIT has a clear effect on melanin-related genes. The results of the present study demonstrate that the mRNA levels of KIT and the melanin-related genes TYR, MITF, PMEL and DCT increased significantly after overexpression of KIT. Conversely, the mRNA levels of these genes clearly decreased after silencing. The results indicate that KIT has a vital role in influencing melanin-related genes at the transcription level.

Melanocyte proliferation and apoptosis are regulated by melanin-related genes and other regulatory interactions. Previous studies have reported that interferon-gamma has a pivotal role in the induction of apoptosis and inhibition of melanogenesis in human melanocytes (Yang et al., 2015). Furthermore, it has been demonstrated that proliferation and melanogenesis in mouse melanocytes can be inhibited by Wnt5a (Zhang et al., 2013). In normal human melanocytes, activation of khellin by ultraviolet $\mathrm{A}$ was able to induce melanocyte proliferation and promote melanogenesis (Carlie et al., 2015). In addition, it has been demonstrated that the expression of KIT tyrosine kinase receptor is critical for melanocyte development in human skin (Grichnik et al., 1996). The numbers of melanocytes expressing KIT protein receptor in the skin of patients with vitiligo is lower, indicating that the expression of KIT is related to melanocytes (Norris et al., 1996). Similar results have shown that overexpression of KIT ligand in the epidermis results in melanocytic hyperplasia in transgenic mice (Kunisada et al. 1998). However, whether the function of KIT in regulating the proliferation and apoptosis of Rex rabbit melanocytes is similar to that of humans or mice remains unclear. The results of the present study indicate that melanocyte proliferation was enhanced and melanocyte apoptosis consistently inhibited by overexpression of KIT, indicating that KIT has a positive effect in melanogenesis. In addition, melanin content increased due to melanogenesis. Therefore, we believe that KIT has an important effect on melanocyte proliferation and apoptosis, further regulating melanogenesis.

\section{Conclusions}

In conclusion, the KIT gene has an important role in regulating melanocyte proliferation and melanogenesis. KIT mRNA and protein expression levels in the skin of Rex rabbit with black coats were higher than in the skin of other colors, indicating that the KIT gene is involved in the process of creating coat colors, and its expression associated with the production of eumelanin. KIT mRNA expression and that of melanin-related genes was substantially affected by a change in KIT expression $(P<0.01)$, revealing that the expression of melanin-related genes was regulated. Furthermore, KIT can promote melanocyte proliferation and inhibit apoptosis. 


\section{Acknowledgements}

277 The study was supported by the National Natural Science Foundation of China (Grant No. 278 31702081), Modern Agricultural Industrial System Special Funding (CARS-43-A-1), and Science 279 and Technology Major Project of New Variety Breeding (Livestock and Poultry) of Zhejiang 280 Province, China (2016C02054-10).

\section{Declaration of interest}

282 The authors declare no conflict of interest.

\section{Data Availability}

284 The following information was supplied regarding data availability:

285 The raw data are provided in the Supplemental Files.

\section{Author contributions}

287 All authors read and approved the manuscript. Shuaishuai $\mathrm{Hu}$ was responsible for the collection, 288 analysis of results, performance of experiments and wrote the manuscript. Yang Chen and Bohao 289 Zhao performed experiments. Naisu Yang, Shi Chen and Jinyu Shen prepared figures and/or 290 tables. Guolian Bao and Xinsheng Wu designed the study. All authors read and approved the final 291 manuscript. 
292

293

294

295

296

297

298

299

300

301

302

303

304

305

306

307

308

309

310

311

312

313

314

315

316

317

318

319

320

321

322

323

324

325

\section{References}

Baxter, L.L., Watkins-Chow, D.E., Pavan, W.J., Loftus, S.K., 2019. A curated gene list for expanding the horizons of pigmentation biology. Pigment Cell Melanoma Research 32, 348358. https://doi.org/10.1111/pcmr.12743.

Bennett, D.C., Lamoreux, M.L, 2003. The color loci of mice-a genetic century. Pigment Cell Melanoma Research 16, 333-344. https://10.1034/j.1600-0749.2003.00067.x.

Brenig, B., Beck, J., Floren, C., 2013. Molecular genetics of coat colour variations in White Galloway and White Park cattle 44, 450-453. https://doi.org/10.1111/age.12029.

Carlie, G., Ntusi, N.B.A., Hulley, P.A., Kidson, S.H., 2015. KUVA (khellin plus ultraviolet A) stimulates proliferation and melanogenesis in normal human melanocytes and melanoma cells in vitro. British Journal of Dermatology 149, 707-717. https://doi.org/10.1046/j.13652133.2003.05577.x.

Chen, T., Zhao, B., Yu, L., Wang, R., Yang, Y., Yang, L., Dong, C.S., 2018. MITF-M Regulates Melanogenesis in Mouse Melanocytes. Journal of Dermatological Science 90, 253-262. https://doi.org/10.1016/j.jdermsci.2018.02.008.

Chen, Y., Hu, S., Mu, L., Zhao, B., Wang, M., Yang, N., Bao, G., Zhu, C., Wu, X.S., 2019. Slc7a11 modulated by POU2F1 is involved in pigmentation in rabbit. International journal of molecular sciences 20, 2493. https://doi.org/10.3390/ijms20102493.

Curtin, J.A., Klaus, B., Daniel, P., Bastian, B.C., 2006. Somatic activation of KIT in distinct subtypes of melanoma. Journal of Clinical Oncology Official Journal of the American Society of Clinical Oncology 24, 4340-4346. https://doi.org/10.1200/JCO.2006.06.2984.

Greg B, George C, 2007. How hair gets its pigment. Cell 130, 779-781. https://doi.org/10.1016/j.cell.2007.08.032.

Garrido, M., Bastian, B.C., 2010. KIT as a therapeutic target in melanoma. Journal of Investigative Dermatology 130, 20-27. https://doi.org/10.1038/jid.2009.334.

Grichnik, J. M. , Ali, W. N. , Burch, J. A. , Byers, J. D. , Garcia, C. A. , \& Clark, R. E. , 1996. Kit expression reveals a population of precursor melanocytes in human skin. Journal of Investigative Dermatology, 106(5), 967-71. https://doi:10.1111/1523-1747.ep12338471.

Grichnik, J.M., 2006. Kit and melanocyte migration. Journal of Investigative Dermatology 126, 945. https://doi.org/10.1038/sj.jid.5700164.

Harris, V.M., 2015. Protein Detection by Simple Western ${ }^{\mathrm{TM}}$ Analysis. Methods in Molecular Biology 1312, 465. https://doi.org/10.1007/978-1-4939-2694-7_47.

Hearing, V., 2011. Determination of Melanin Synthetic Pathways. Journal of Investigative Dermatology 131, E8-E11. https://doi.org/10.1038/skinbio.2011.4. 
326

327

328

329

330

331

332

333

334

335

336

337

338

339

340

341

342

343

344

345

346

347

348

349

350

351

352

353

354

355

356

357

358

359

360

Hirobe, T., Wakamatsu, K., Ito, 2007. The eumelanin and pheomelanin contents in dorsal hairs of female recessive yellow mice are greater than in male. Journal of Dermatological Science 45, 55-62. https://doi.org/10.1016/j.jdermsci.2006.10.004.

Holl, H., Isaza, R., Mohamoud, Y., Ahmed, A., Almathen, F., Youcef, C., Gaouar, S., Antczak, D., Brooks, S.J.G., 2017. A frameshift mutation in KIT is associated with white spotting in the Arabian camel. Genes 8, 102. https://doi.org/10.3390/genes8030102.

Ito, S., Miyake, S., Maruyama, S., Suzuki, I., Commo, S., Nakanishi, Y., Wakamatsu, K.J., 2017. Acid hydrolysis reveals a low but constant level of pheomelanin in human black to brown hair. Pigment cell \& melanoma research 31, 393-403. https://doi.org/10.1111/pcmr.12673.

Ito, S., Wakamatsu, K., Ozeki, H.J.P.C., Research, M., 2010. Chemical analysis of melanins and its application to the study of the regulation of melanogenesis. Pigment Cell Research 13, 103-109. https://doi.org/10.1034/j.1600-0749.13.s8.19.x.

Ito, S, 2010. A Chemist's View of Melanogenesis. Pigment Cell \& Melanoma Research 16, 230 236. https://doi.org/10.1034/j.1600-0749.2003.00037.x.

Ji, H.H., Jeong, Y.J., Xuan, S.H., Lee, J.Y., Park, J., Park, S.N., 2018. Methyl-2-acetylamino-3(4-hydroxyl-3,5-dimethoxybenzoylthio)propanoate suppresses melanogenesis through ERK signaling pathway mediated MITF proteasomal degradation. Journal of Dermatological Science 91, 142-152. https://doi.org/10.1016/j.jdermsci.2018.04.011.

Lan W J, Wang H Y, Lan W, Wang K Y, 2008. Geniposide Enhances Melanogenesis by Stem Cell Factor/c-Kit Signalling in Norepinephrine-Exposed Normal Human Epidermal Melanocyte. Basic \& Clinical Pharmacology \& Toxicology, 103, 88-93. https:/doi: 10.1111/j.17427843.2008.00251.x.

Li, J., Jiang, Q., Chen, W., Li, Y., Jiang, H., Huo, J., Zhang, Q., 2017. Differential Expression of the KIT Gene in Liaoning Cashmere Goats with different Coat Colors. Pakistan Journal of Zoology 49, 2299-2305. http://dx.doi.org/10.17582/journal.pjz/2017.49.6.2299.2305

Kunisada, T. , Lu, S. Z. , Yoshida, H. , Nishikawa, S. , Nishikawa, S. I. , \& Mizoguchi, M. , 1998. Murine cutaneous mastocytosis and epidermal melanocytosis induced by keratinocyte expression of transgenic stem cell factor. Journal of Experimental Medicine, 187(10), 15651573. http://10.1084/jem.187.10.1565

Mizutani, Y., Hayashi, N.M., Imokawa, G., 2010. A single UVB exposure increases the expression of functional KIT in human melanocytes by up-regulating MITF expression through the phosphorylation of p38/CREB. Archives of dermatological research 302, 283-294. https://doi.org/10.1007/s00403-009-1007-x.

Mochly-Rosen, D., 1995. Localization of protein kinases by anchoring proteins: a theme in signal transduction. Science 268, 247-251. https://doi.org/10.1126/science.7716516. 
361

362

363

364

365

366

367

368

369

370

371

372

373

374

375

376

377

378

379

380

381

382

383

384

385

386

387

388

389

390

391

392

393

394

395

Nicola, S., Maria, R., Diego, R., Celine, T., Roberto, A., Manuela, C., Luca, C., Kazumasa, W., 2013. Sexual dimorphism in melanin pigmentation, feather coloration and its heritability in the barn swallow (Hirundo rustica). PLoS One 8, e58024. https://doi.org/10.1371/journal.pone.0058024.

Norris, A., Todd, C., Graham, A., Quinn, A. G., \& Thody, A. J, 1996. The expression of the c-kit receptor by epidermal melanocytes may be reduced in vitiligo. British Journal of Dermatology, 134(2), 299-306. https://doi.org/10.1111/j.1365-2133.1996.tb07618.x.

Oiso, N., Wakamatsu, K., Yanagihara, S., Kawada, A., 2018. Nevus depigmentosus with yellow hair colour due to an excess amount of benzothiazine-type pheomelanin. European Journal of Dermatology 28, 126-128. https://doi.org/10.1684/ejd.2017.3193.

Oiso, N., Wakamatsu, K., Yanagihara, S., Kawada, A., 2018. Decreased benzothiazole-type phemelanin in regrown brown hair in alopecia areata. European Journal of Dermatology 28, 130-131. https://doi.org/10.1684/ejd.2017.3194.

Reiko, K., Katsuhiko, T., Kazutoshi, H., Akira, S., Shinji, S., Takeshi, K., Genji, I., 2004. Mechanisms underlying the dysfunction of melanocytes in vitiligo epidermis: role of $\mathrm{SCF} / \mathrm{KIT}$ protein interactions and the downstream effector, MITF-M. Journal of Pathology 202, 463. https://doi.org/10.1002/path.1538.

Sakaizawa, K., Ashida, A., Uchiyama, A., Ito, T., Fujisawa, Y., Dai, O., Matsushita, S., Fujii, K., Fukushima, S., Shibayama, Y.J., 2015. Clinical characteristics associated with BRAF, NRAS and KIT mutations in Japanese melanoma patients. Journal of dermatological science 80, 33-37. https://doi.org/10.1016/j.jdermsci.2015.07.012.

Schmittgen, T.D., Livak, K.J.J., 2008. Analyzing real-time PCR data by the comparative C T method. Nature protocols 3, 1101. https://doi.org/10.1038/nprot.2008.73.

Shosuke, I., Kazumasa, W.J.P., Photobiology, 2010. Chemistry of mixed melanogenesis--pivotal roles of dopaquinone. Photochemistry and photobiology 84, 582-592. https://doi.org/10.1111/j.1751-1097.2007.00238.x.

Solomon L M , Witkop C., 1980. The Coat Colors of Mice (Book). Journal of Investigative Dermatology 74, 445-445.

Song, X., Xu, C., Liu, Z., Yue, Z., Liu, L., Yang, T., Cong, B., Yang, F., 2017. Comparative Transcriptome Analysis of Mink (Neovison vison) Skin Reveals the Key Genes Involved in the Melanogenesis of Black and White Coat Colour. Scientific reports 7, 12461. https://10.1038/s41598-017-12754-0.

Sturm, R.A., Teasdale, R.D., Box, N.F., 2001. Human pigmentation genes: identification, structure and consequences of polymorphic variation. Gene 277, 49-62. https://doi.org/10.1016/S0378-1119(01)00694-1. 
396

397

398

399

400

401

402

403

404

405

406

407

408

409

410

411

412

413

414

415

416

417

418
Tamate, H.B., Takeuchi, T.J.G., 2010. Induction of the shift in melanin synthesis in lethal yellow (Ay/a) mice in vitro. Developmental Genetics 2, 349-356. https://doi.org/10.1002/dvg.1020020404.

Wen, B., Chen, Y., Li, H., Wang, J., Shen, J., Ma, A., Qu, J., Bismuth, K., Debbache, J., Arnheiter, H.J., 2010. Allele-specific genetic interactions between Mitf and Kit affect melanocyte development. Pigment cell \& melanoma research 23, 441-447. https://doi.org/10.1111/j.1755-148X.2010.00699.x.

Yang, L., Wei, Y., Sun, Y., Shi, W., Yang, J., Zhu, L., Li, M.J., 2015. Interferon-gamma Inhibits Melanogenesis and Induces Apoptosis in Melanocytes: A Pivotal Role of CD8+ Cytotoxic $\mathrm{T}$ Lymphocytes in Vitiligo. Acta dermato-venereologica 95, 664-671. https://doi.org/10.2340/00015555-2080.

Yasumoto, K., Yokoyama, K., Takahashi, K., Tomita, Y., Shibahara, S., 1997. Functional analysis of microphthalmia-associated transcription factor in pigment cell-specific transcription of the human tyrosinase family genes. Journal of Biological Chemistry 272, 503-509. https://doi.org/10.1074/jbc.272.1.503.

Zhang, J., Li, Y., Wu, Y., Yang, T., Yang, K., Wang, R., Yang, J., Guo, H.J., 2013. Wnt5a inhibits the proliferation and melanogenesis of melanocytes. International journal of medical sciences 10, 699-706. https://doi.org/10.7150/ijms.5664.

Zhao, B., Chen, Y., Yang, N., Chen, Q., Bao, Z., Liu, M., Hu, S., Li, J., Wu, X.S., 2019. miR-218-5p regulates skin and hair follicle development through $\mathrm{Wnt} / \beta$-catenin signaling pathway by targeting SFRP2. Journal of Cellular Physiology. https://doi.org/10.1002/jcp.28633. 
419 Figure legends

420 Figure 1. The Rex rabbits of different coat colors. (a): White, (b): Black, (c): Chinchilla, (d): 421 Brown, (e): Gray, (f): Gray-yellow.

422 Figure 2. Cloning of the KIT gene and mRNA expression in Rex rabbits with different coat colors. 423 (a)(b)(c) 5'UTR , 3'UTR and cDNA of KIT was acquired by RACE and cloning techniques. (d) 424 Neighbor-joining phylogenetic tree of Oryctolagus cuniculus and other species based on KIT 425 cDNA. (e) mRNA expression of KIT gene in rabbits of different coat colors by qPCR. The 426 expression levels of KIT in the Black, Chinchilla, Brown, Gray, and Gray-yellow color group were 427 normalized to the White color group. Error bars indicate the mean \pm SD of four repeated 428 experiments. (f) KIT protein expression in rabbits of different coat colors was detected by the Wes method. WH: White, BL: Black, CH: Chinchilla, BR: Brown, GR: Gray, GY: Gray-yellow. (g)

430

431

432

433

434

435

436

437

438

439

440

441

442

443

444

445

446

447

448

449

450

451 the relative expression ratio of KIT protein in rabbits of different coat colors were quantitatively calculated by the Compass program.

Figure 3. mRNA expression of melanogenesis-related genes was regulated by the change of KIT expression. (a) The conserved domain of KIT was predicted by NCBI. (b) KIT mRNA expression levels was detected by qPCR after transfection with siRNAs-KIT. (c) mRNA expression of melanogenesis-related genes was analyzed by qPCR after overexpression of KIT. (d) The protein expression levels of KIT was detected by qPCR when KIT was overexpressed or knocked down. (e) The protein results were quantitatively analyzed using the Compass program and the relative expression ratio of KIT was calculated. (f) mRNA expression levels of melanogenesis-related genes was detected by qPCR when KIT was overexpressed. ${ }^{* *} P<0.01,{ }^{*} P<0.05$. (g) mRNA expression levels of melanogenesis-related genes was detected by qPCR after knockdown of KIT. ${ }^{* *} P<0.01,{ }^{*} P<0.05$.

Figure 4. Melanogenesis, melanocyte proliferation and apoptosis were controlled by the change of KIT expression. (a) $48 \mathrm{~h}$ after transfection with overexpression of KIT, melanin content was analyzed by $\mathrm{NaOH}$ lysis. (b) $48 \mathrm{~h}$ after transfection with knockdown of KIT, melanin content was analyzed by $\mathrm{NaOH}$ lysis. (c) Melanocyte proliferation was estimated by CCK-8 assay 24,48 , and $72 \mathrm{~h}$ after overexpression of KIT. (d) Melanocyte proliferation was estimated by CCK-8 assay 24, 48, and $72 \mathrm{~h}$ after knockdown of KIT. (e) Cell apoptosis in melanocyte was detected after overexpression of KIT. (f) Cell apoptosis rate in melanocyte was calculated after overexpression of KIT. ${ }^{* *} P<0.01,{ }^{*} P<0.05$. (g) Cell apoptosis in melanocyte was detected after knockdown of KIT. (h) Cell apoptosis rate in melanocyte was calculated after knockdown of KIT. ${ }^{* *} P<0.01,{ }^{*} P$ $<0.05$. 


\section{Figure 1}

The Rex rabbits of different coat colors.

(a): White, (b): Black, (c): Chinchilla, (d): Brown, (e): Gray, (f): Gray-yellow.

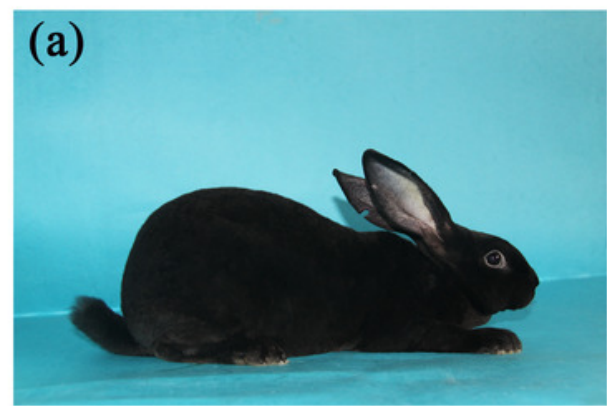

(d)

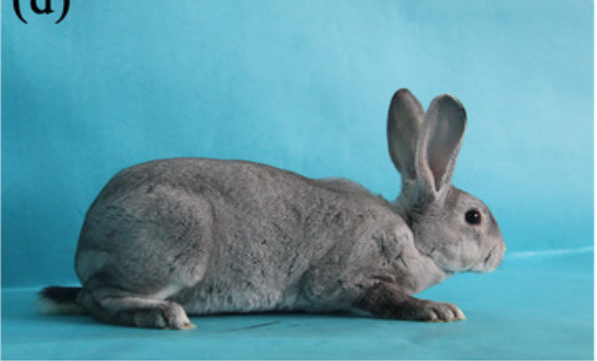

(b)

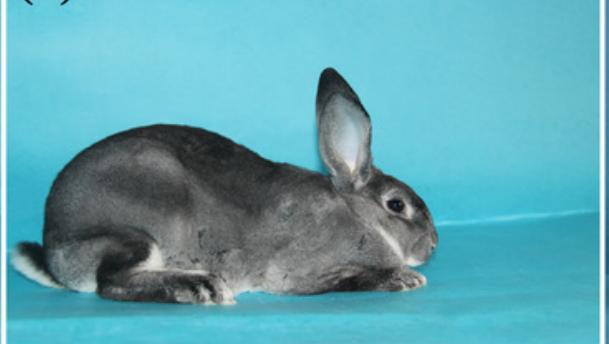

(e)

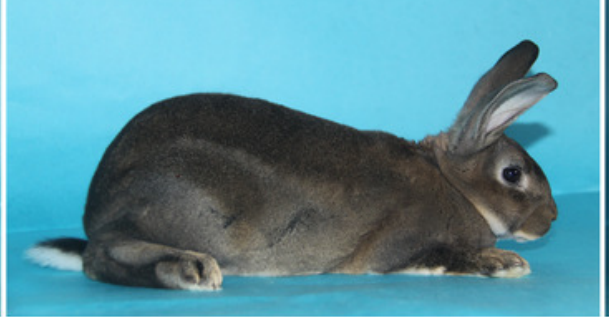

(c)

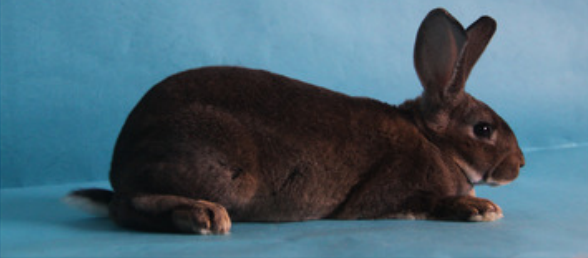

(f) 


\section{Figure 2}

Cloning of the KIT gene and mRNA expression in Rex rabbits with different coat colors.

(a)(b)(c) 5'UTR , 3'UTR and CDNA of KIT was acquired by RACE and cloning techniques. (d) Neighbor-joining phylogenetic tree of Oryctolagus cuniculus and other species based on KIT CDNA. (e) mRNA expression of KIT gene in rabbits of different coat colors by qPCR. (f) KIT protein expression in rabbits of different coat colors was detected by Western Blotting. Small letters indicate significant differences among groups $(P<0.05)$. WH: White, BL: Black, $\mathrm{CH}$ : Chinchilla, BR: Brown, GR: Gray, GY: Gray-yellow.

(a)

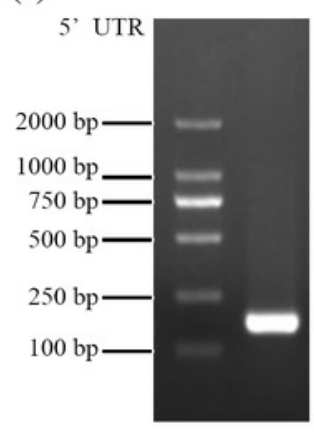

(e)

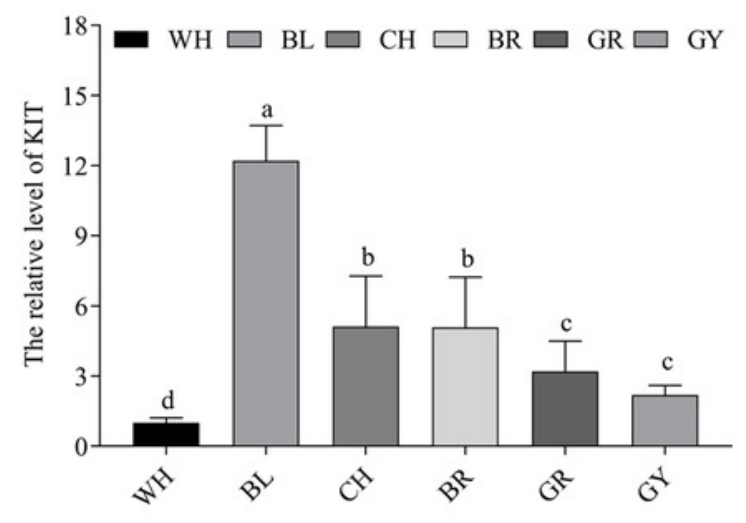

(b)

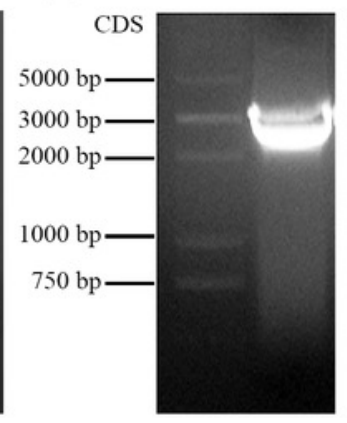

(c)

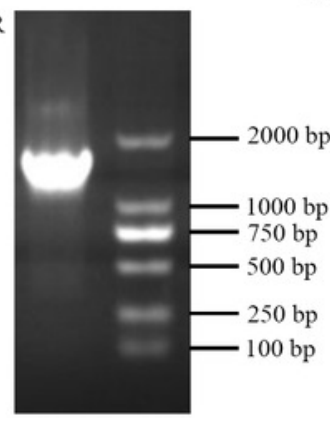

(d)

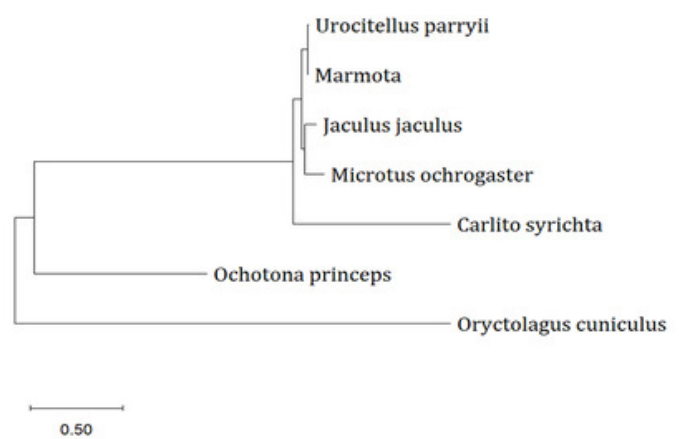

(f)

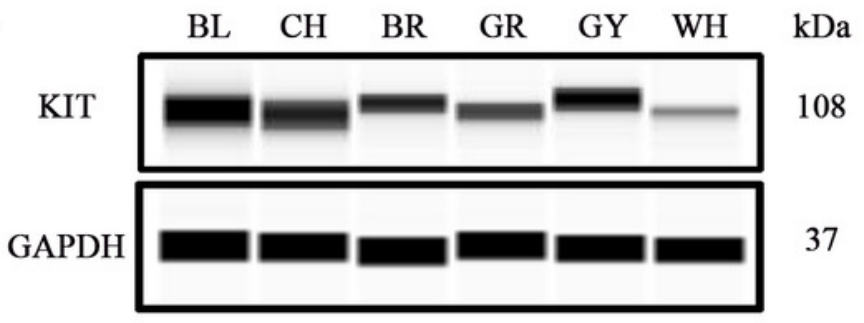

(g)

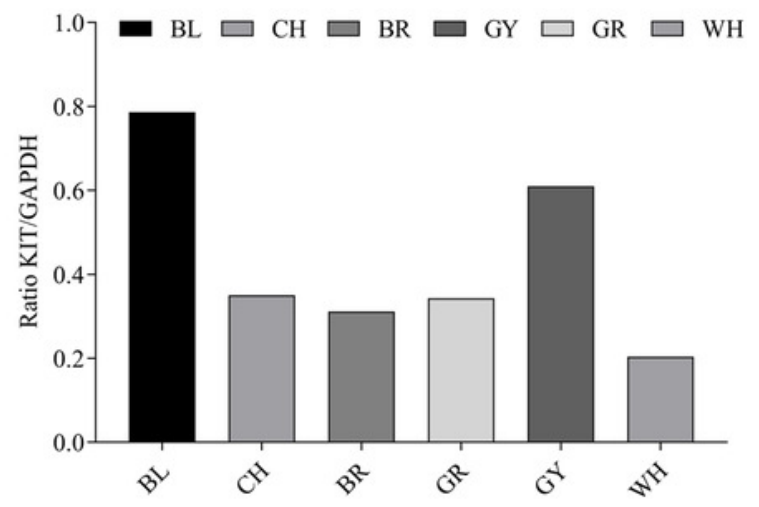




\section{Figure 3}

mRNA expression of melanogenesis-related genes was regulated by the change of KIT expression.

(a) The conserved domain of KIT was predicted by NCBI. (b) KIT mRNA expression levels was detected by qPCR after transfection with siRNAs-KIT. (c) mRNA expression of melanogenesisrelated genes was analyzed by qPCR after knockdown of KIT. (d) mRNA expression levels of KIT was detected by qPCR when KIT was overexpressed. (e) mRNA expression levels of melanogenesis-related genes as detected by qPCR when KIT was overexpressed. ${ }^{* * P}<0.01$, $* P<0.05$. 
(a)

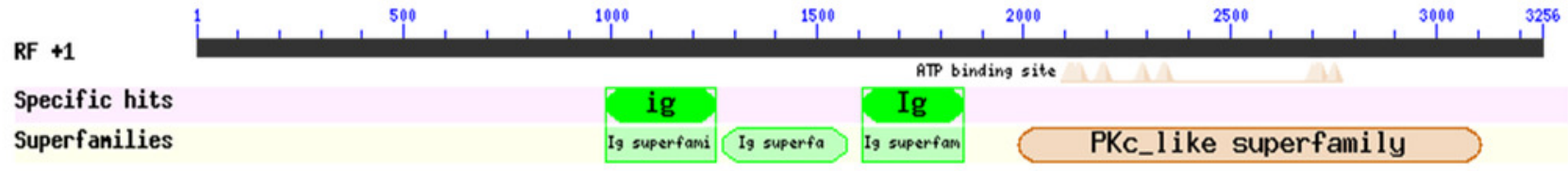

(b)

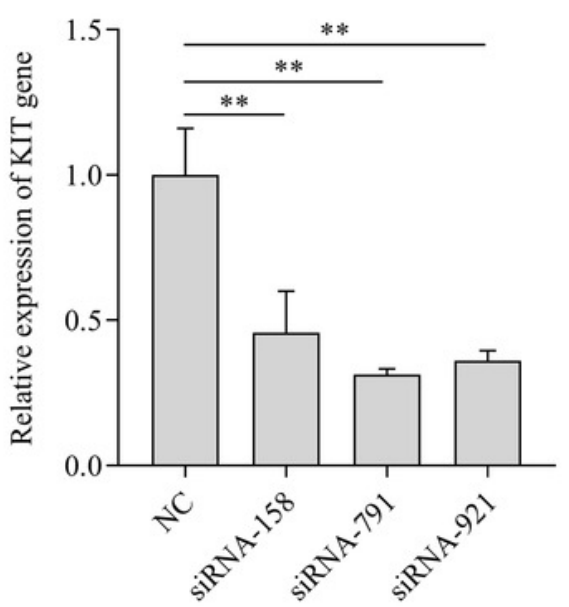

(d)

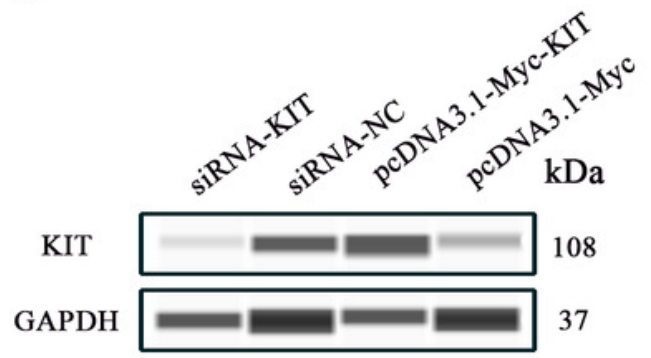

(f)

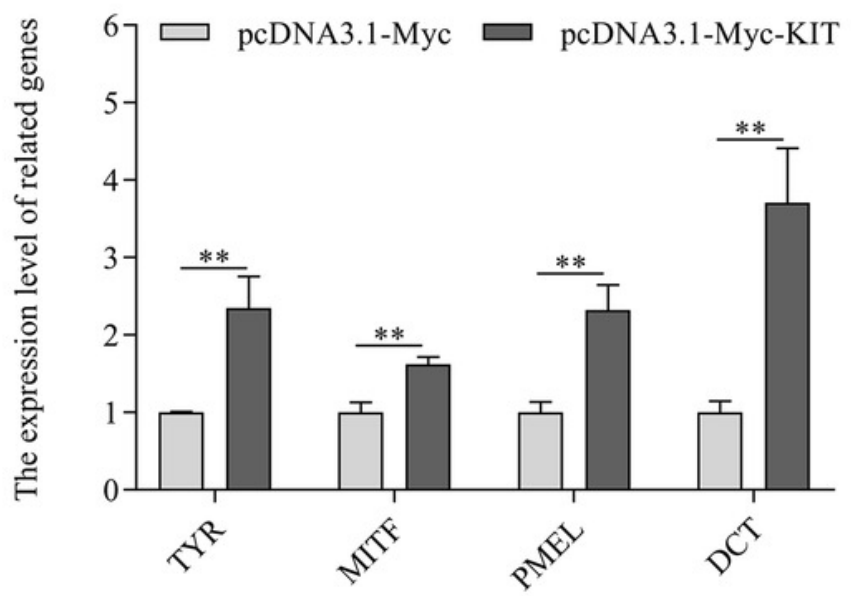

(e) (c)
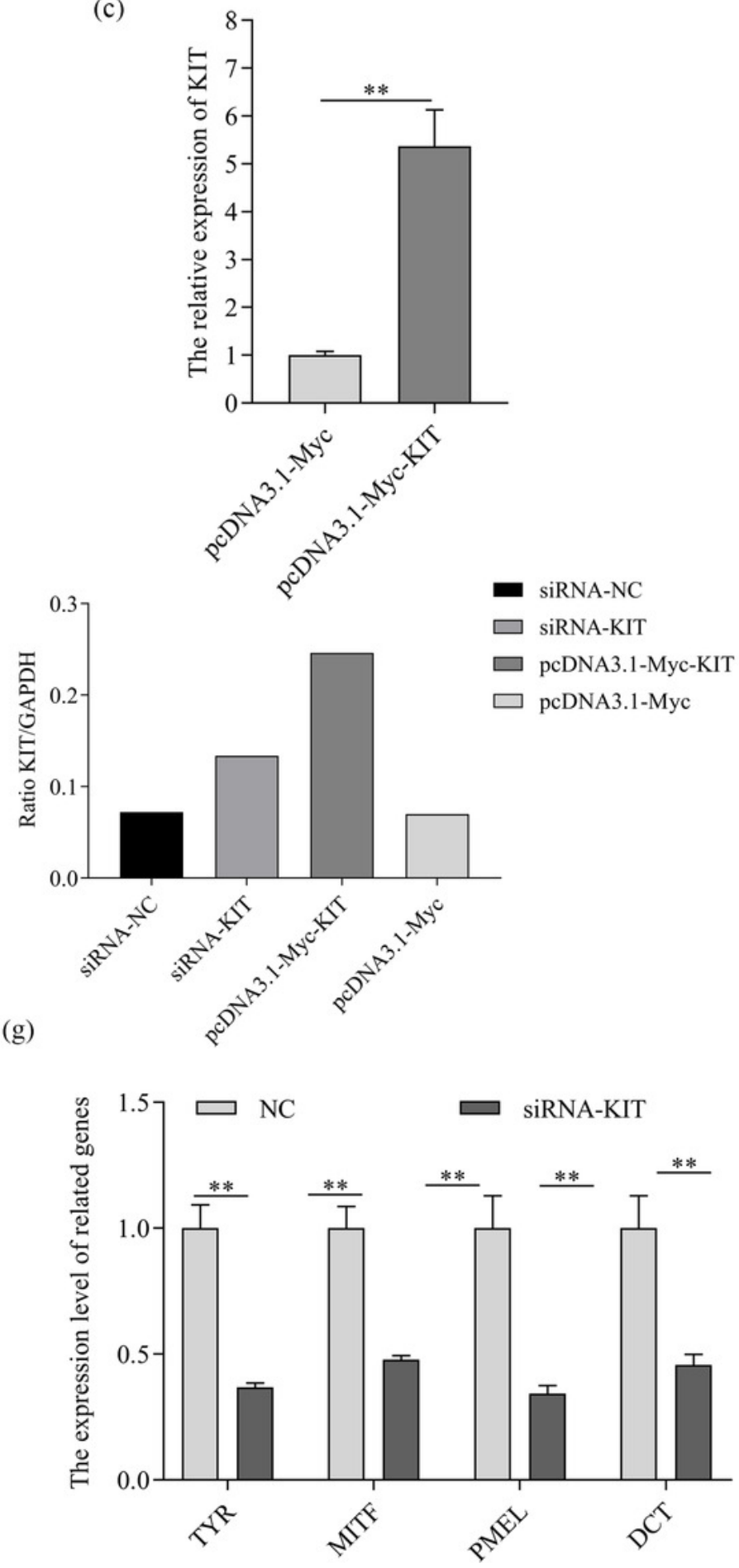


\section{Figure 4}

Melanogenesis, melanocyte proliferation and apoptosis were controlled by the change of KIT expression.

(a)(b) $48 \mathrm{~h}$ after transfection with overexpression or knockdown of KIT, melanin content was analyzed by $\mathrm{NaOH}$ lysis. (c)(d) Melanocyte proliferation was estimated by CCK-8 assay 24, 48, and $72 \mathrm{~h}$ after overexpression or knockdown of KIT. (e)(f) Cell apoptosis rate in melanocyte was assessed after overexpression or knockdown of KIT. ${ }^{* * P}<0.01,{ }^{*} P<0.05$. 
(a)

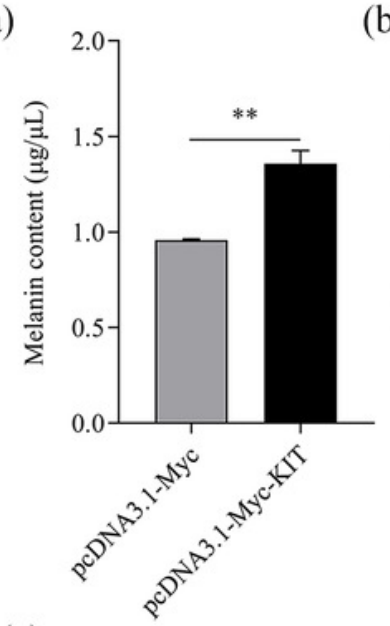

(e)
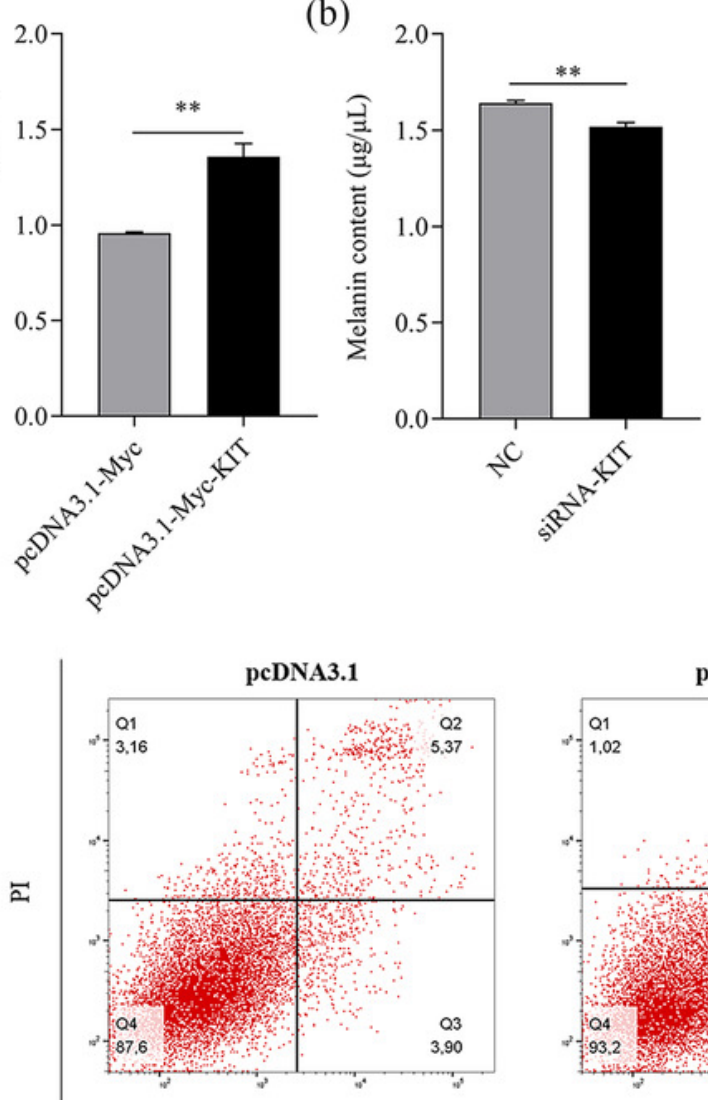

(c)

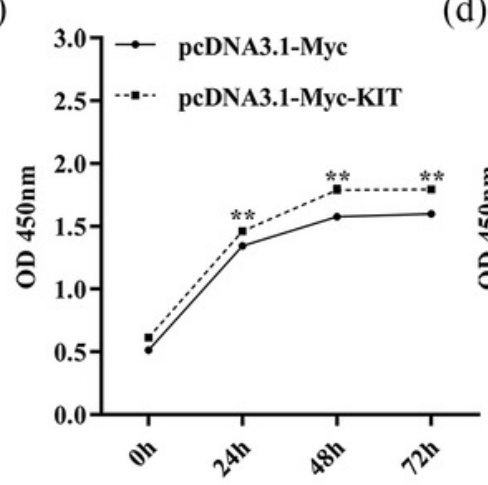

(d)

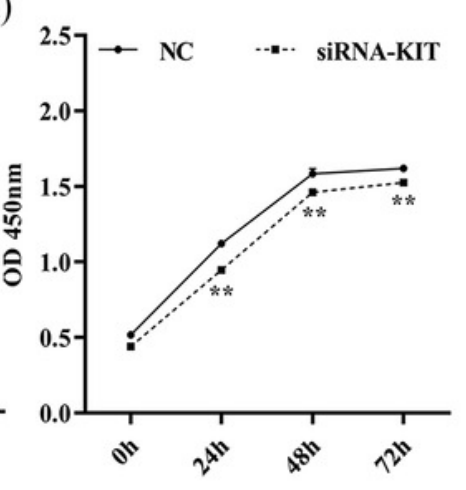

(g)

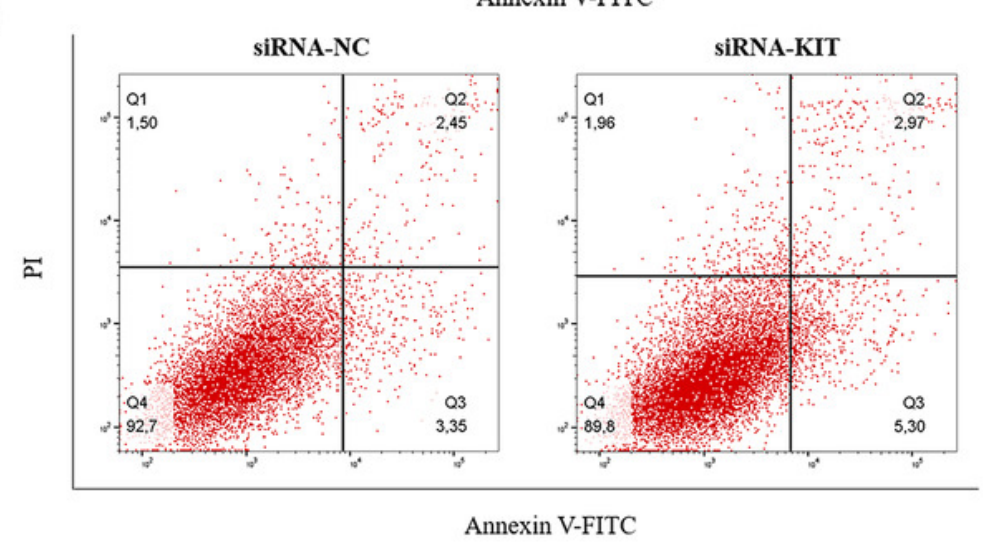

(f)

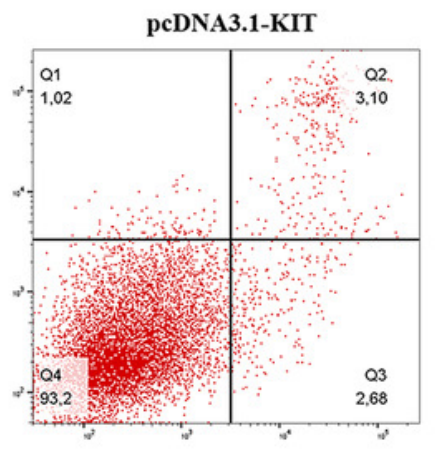

(h)
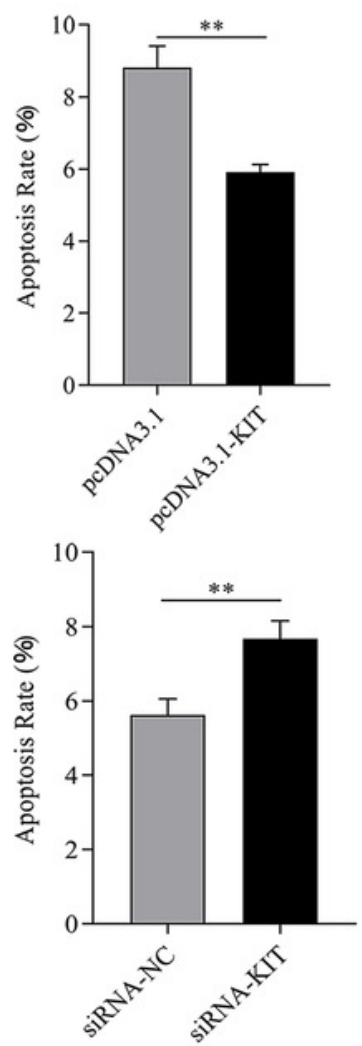


\section{Table 1 (on next page)}

Primers used in the KIT RACE assay

Primers for three specific 5' untranslated regions (UTRs), coding sequence ( CDS ), and two 3' UTRs of KIT were synthesized. 
1 Table 1 Primers used in the KIT RACE assay

\begin{tabular}{clc}
\hline Name & Sequence $\left(5^{\prime}\right.$ to $\left.3^{\prime}\right)$ & Experiment \\
\hline B065-1 (GSP1) & GGAGTTTCCCAGGAGTCGGC & 5' RACE \\
B065-2 (GSP2) & GCTCGGTTTCAGCATCTTCACA & 5' RACE \\
B065-3 (GSP3) & GCCTTGGAACTGGGACTGAG & 5' RACE \\
C047-1 & CCGACGGCTGCTTGTTTTAG & 3' RACE \\
C047-2 & CTCTTCTTGTTGCTGTGGG & 3' RACE \\
KIT-R & ATGCTTTTTTTCCGCCAAAGA & CDS \\
KIT-F & GTCTTCATGCACGAGCAGGG & \\
\hline
\end{tabular}

2 


\section{Table 2 (on next page)}

Primer sequences of siRNA-KIT

siRNAs of KIT and the negative control were purchased from Shanghai GenePharma Co., Ltd. 
1 Table 2 Primer sequences of siRNA-KIT.

\begin{tabular}{ll}
\hline Name & Sequence $\left(5^{\prime}\right.$ to $\left.3^{\prime}\right)$ \\
\hline Negative Control & Forward: UUCUCCGAACGUGUCACGUTT \\
& Reverse: ACGUGACACGUUCGGAGAATT \\
siRNA-158 & Forward: GGUUCUCGCUGGAGUGCAUTT \\
& Reverse: AUGCACUCCAGCGAGAACCTT \\
siRNA-791 & Forward: GCUGGCAUCAGGGCGACUUTT \\
& Reverse: AAGUCGCCCUGAUGCCAGCTT \\
siRNA-921 & Forward: CCUUGAAAGUCGUAGAUAATT \\
& Reverse: UUAUCUACGACUUUCAAGGTT
\end{tabular}

2 


\section{Table 3(on next page)}

Primer sequences for quantitative real-time PCR

KIT, MITF, TYR, DCT, PMEL and GAPDH gene sequences were obtained from NCBI for design of the primers. 
1 Table 3 Primer sequences for quantitative real-time PCR.

\begin{tabular}{|c|c|c|}
\hline Genes & Sequence $\left(5^{\prime}\right.$ to $\left.3^{\prime}\right)$ & Product length (bp) \\
\hline \multirow{3}{*}{ KIT } & Forward: GGAGTTTCCCAGGAGTCGGC & \multirow{3}{*}{139} \\
\hline & & \\
\hline & Reverse: GCTCGGTTTCAGCATCTTCACA & \\
\hline \multirow{3}{*}{ MITF } & Forward: GCCTTGGAACTGGGACTGAG & \multirow{3}{*}{142} \\
\hline & & \\
\hline & Reverse: CCGACGGCTGCTTGTTTTAG & \\
\hline \multirow{3}{*}{ TYR } & Forward: CTCTTCTTGTTGCTGTGGG & \multirow{3}{*}{156} \\
\hline & & \\
\hline & Reverse: GCTGAGTAGGTTAGGGTTTTC & \\
\hline \multirow{3}{*}{$\mathrm{DCT}$} & Forward: ATTCTGCTGCCAATGACCC & \multirow{3}{*}{154} \\
\hline & & \\
\hline & Reverse: AACGGCACCATGTTATACCTG & \\
\hline \multirow{3}{*}{ PMEL } & Forward: GTCAGCACCCAGCTTGTCA & \multirow{3}{*}{130} \\
\hline & & \\
\hline & Reverse: GCTTCATTAGTCTGCGCCTGT & \\
\hline \multirow{3}{*}{ GAPDH } & Forward: CACCAGGGCTGCTTTTAACTCT & \multirow{3}{*}{141} \\
\hline & & \\
\hline & Reverse: CTTCCCGTTCTCAGCCTTGACC & \\
\hline
\end{tabular}




\section{Table 4(on next page)}

Table 4. Correlation between gene expression and melanin content.

the correlation analysis between mRNA expression of KIT and melanin content was performed. 
1 Table 4. Correlation between gene expression and melanin content.

\begin{tabular}{lcc}
\hline Gene expression & KIT overexpression & KIT knockdown \\
\hline \multicolumn{1}{c}{ Melanin content $(\mu \mathrm{g} / \mu \mathrm{L})$} & $1.000^{* *}$ & $1.000^{* *}$ \\
\hline $\begin{array}{l}\text { Significant difference is denoted by }{ }^{*} P<0.05 \\
<0.01 \text {; extremely significant difference is denoted by }{ }^{* *} P\end{array}$
\end{tabular}

$3<0.01$. 